\title{
Robust Adaptive Backstepping Controller for Altitude Control of a Small Scale Helicopter by Considering the Ground Effect Compensation
}

\author{
Tushar K. Roy \\ Department of ETE, RUET, Bangladesh \\ *Corresponding author: roy_kanti@yahoo.com
}

Copyright (C) 2013 Horizon Research Publishing All rights reserved.

\begin{abstract}
In this paper, I focus on the design and implementation of a controller for a two degree-of-freedom system. A nonlinear robust adaptive control technique is proposed to control the altitude of a small-scale helicopter for hovering as well as vertically take-off/landing near the ground surface in the presence of strong horizontal wind gusts. In order to stabilize the vertical dynamics of the small-scale helicopter, a recursive (backstepping) design procedure is used to design the robust adaptive controller based on Lyapunov approach. Simulation results demonstrate that the proposed robust adaptive backstepping controller is capable of controlling the altitude for hovering flight of a small-scale helicopter near ground surface in the presence of strong horizontal wind gusts.
\end{abstract}

Keywords Robust Adaptive Backstepping Control, Ground Effect, Lyapunov Function, UAH

\section{Introduction}

Among the variety of Unmanned Air Vehicles (UAVs), unmanned autonomous helicopters constitute one of the most versatile and agile platforms. A helicopter can operate in different flight modes, such as vertical take-off/landing, hovering, longitudinal/lateral flight, and bank to turn which gives them the advantage of effective observation from various positions. Among these abilities hovering and vertical take-off is necessarily needed. Unmanned autonomous helicopter control system should make these performances achieved by improving the tracking performance and disturbance rejection capability in different weather conditions. Comparing with the fixed wing aircraft, that of helicopter altitude is much lower and close to the ground; so ground effect is the important topic [1]. Ground effect is most significant in hover, and below the heights of the order of a rotor radius. Ground effect usually supports the additional lift of the helicopter, so it should be considered in the automatic control system design because precise altitude control is critical for a small helicopter to avoid rapid uplift and to make a soft landing. It should be noted that ground effect also depends on the wind gusts and the hardness of the ground surface. Wind gusts and ground effect can create large thrust variations in the helicopter, which create a challenge to maintain a constant altitude near ground surface. Control of a model scale helicopter under vertical wind gusts is discussed in [2]. The typical level of wind gusts in this paper is less than $1 \mathrm{~m} / \mathrm{s}$. Furthermore, the authors present an active disturbance-rejection control strategy based on a nonlinear observer. In [3], the authors propose a feedback-feedforward proportional differential (PD) controller to stabilize the vertical dynamics of a small helicopter in the presence of the horizontal wind gusts. A gust estimator is developed to obtain the information on wind gust levels in the presence of sensor errors including accelerometer vibration, accelerometer drift and measurement error of vertical velocity. The estimation of the wind gusts is then fed to a feedback-feedforward proportional derivative controller to compensate the effects of the horizontal gusts in the stabilization of helicopter altitude. The position control of a small scale unmanned autonomous helicopter under the vertical and horizontal wind gusts using the feedback method is addressed in [4], wherein the wind disturbance is assumed to be the sum of a fixed number of sinusoids with unknown amplitudes, frequencies and phases. In [5], the authors designed a multi-mode linear control strategy for unmanned autonomous helicopter in the presence of model uncertainties, atmospheric disturbances and by considering the handling qualities requirements. A robust backstepping controller to control of a small scale model autonomous helicopter in the presence of parametric uncertainties as well as uniform time varying three-dimensional wind gusts is addressed in [6]. With the assistance of an unknown input observer technique (UIO), the controller is reported to be able to handle the effect of these uncertainties on the autonomous helicopter. For altitude control, [7] uses 
adaptive control, while [8] uses neural network based control, to deal with both unmodeled dynamics and aerodynamic disturbance from the environment. Even though the helicopter altitude control problem is successfully resolved in $[9,11]$, but the effects of wind disturbances are not taken into account within the system model. For altitude control near the ground surface, a sliding mode controller is proposed in [12], in which a constant wind disturbance is used.

It should be emphasized that very few research works in the literature, are taking into account the ground effect and external wind gusts in the altitude control of a small helicopter near ground surface. In the previous version of this paper [13], the authors designed a robust backstepping controller for altitude control of a small helicopter by considering both the effects of ground effect and external wind gusts but in $[15,16,17]$ the author did not consider the ground effect. Throughout this paper, for an unmanned autonomous helicopter altitude control near the ground surface, both ground effect compensation and external wind gusts are taken into account within the system model. However, in our case, we concentrate on horizontal wind gusts with a typical level of $10 \mathrm{~m} / \mathrm{s}$. Vertical wind gusts can be neglected compared with its horizontal counterparts since the main factor influencing thrust in hover comes from the horizontal gusts, particularly close to the ground where the vertical gust component is near zero [3].

The main contribution of this paper is to control the altitude of an unmanned small helicopter which is especially important, during takeoff and landing. Lyapunov technique is used to show the robustness of the proposed method under the consideration of wind gusts and ground effect. The performance of the controller is simulated by keeping the altitude constant in the presence of external wind gusts and ground effect near ground surface.

The rest of this paper is organized as follows. Section II briefly introduces the mathematical model which is used in Section IV for the robust adaptive control design. Section III presents the gusts model. Section IV presents the control structure. Section V discusses simulation results. Finally, the paper is concluded in Section VI.

\section{Vertical Dynamics of UAH}

Unmanned Autonomous Helicopter (UAH) has specific characteristics: it can move vertically, float in the air, turn in place, move forward and lateral and can perform these movements in combinations. Because of this, helicopter dynamics modelling is a very complex problem. In 6-DOF form, the motion state and control inputs are represented as

$$
\begin{aligned}
x & =\{u, w, q, \theta, v, p, r, \phi\} \\
u_{c} & =\left\{\delta_{\text {lon }}, \delta_{\text {lat }}, \delta_{\text {col }}, \delta_{\text {ped }}\right\},
\end{aligned}
$$

where, $u, v$ and $w$ represent the linear velocity in the body frame; $p, q$ and $r$ denote roll, pitch and yaw rates, respectively; and $\phi, \theta$ represent roll, pitch attitude, respectively. A conventional single main rotor helicopter has four independent control inputs, $\delta_{\text {lat }}, \delta_{\text {lon }}, \delta_{c o l}$ and $\delta_{p e d}$ which denote the deflection of the lateral cyclic, longitudinal cyclic, main rotor collective pitch and tail rotor collective pitch, respectively. The collective commands control the magnitude of the main rotor and tail rotor thrust and other two control commands control the inclination of the Tip-Path-Plane (TPP) on the longitudinal and lateral direction.

Before getting into the control law design using robust adaptive backstepping technique vertical dynamics should be analysed. Consider the nonlinear form of helicopter equations of motion as follows,

$$
\begin{gathered}
\dot{u}=v r-w q+g \sin \theta+\frac{X}{m} \\
\dot{v}=w p-u r+g \sin \phi \cos \theta+\frac{Y}{m} \\
\dot{w}=(u q-v p)+g \cos \phi \cos \theta+\frac{Z}{m} \\
\dot{p}=q r\left(I_{y y}-I_{z z}\right) / I_{x x}+L / I_{x x} \\
\dot{q}=p r\left(I_{z z}-I_{x x}\right) / I_{y y}+M / I_{y y} \\
\dot{r}=p q\left(I_{x x}-I_{y y}\right) / I_{z z}+N / I_{z z}
\end{gathered}
$$

where, forces $[X, Y, Z]^{T}$ and moments $[L, M, N]^{T}$ are expressed in the body frame, $m$ is the helicopter mass and $I_{x x}$, $I_{y y}$ and $I_{z z}$ are the moment of inertial about $x, y$, and $z$ axis, respectively. Note that these equations are expressed in body frame. Since we are only interested to control the altitude so, we need to express the equation (3) i.e., vertical dynamics in earth frame. To this end, we use the rotation matrix between the body and earth frames and obtain the following vertical dynamics,

$$
\dot{w}=g+(\cos \phi \cos \theta) Z / m
$$

Vertical dynamics can be linearized around hover flight conditions, i.e., $\phi \approx 0, \theta \approx 0$, and $\psi \approx 0$. So, resulting linearized vertical dynamics can be expressed in the earth frame as

$$
\dot{w}=\frac{m g-T_{m r}}{m}
$$

where, $T_{m r}$ is the main rotor thrust of the helicopter.

\section{Gust MODEL}

For analysis of RUAV, wind gusts can be treated as either random (spectral turbulence) or discrete. For random gusts, typical spectral models include the Von Karman and Dryden 
turbulence models. The Von Karman model has been widely considered the more "realistic" model when it comes to defining turbulence spectra. However, due to the computational complexity of the Von Karman model, the Dryden model is typically used in aerospace vehicle analyses. There are many sources for wind models based upon empirical data that consist of passing band limited white noise through appropriate forming filters. The turbulence models are scaled with respect to RUAV altitude, velocity, wing span. Vertical wind gusts can be neglected compared with its horizontal counterparts since the main factor influencing thrust in hover comes from the horizontal gusts, particularly close to the ground where the vertical gust component is near zero. Consequently, this paper consider the horizontal wind gusts model and corresponding forming filters including $H_{u}(s)$ for longitudinal direction and $H_{v}(s)$ for lateral direction, take the following transfer function forms,[13] respectively:

$$
\begin{aligned}
& H_{u}(s)=\sigma_{u} \sqrt{\frac{2 L_{u}}{\pi U}} \frac{1}{1+\frac{L_{u}}{U} s} \\
& H_{v}(s)=\sigma_{v} \sqrt{\frac{L_{v}}{\pi U}} \frac{1+\frac{\sqrt{3} L_{v}}{U} s}{\left(1+\frac{L_{v}}{U} s\right)^{2}}
\end{aligned}
$$

where, $U$ is the true speed of a RUAV, $\sigma_{u}$, and $\sigma_{v}$ are the root mean square intensities of the turbulence and $L_{u}$, and $L_{v}$ are the turbulence scale lengths that describe the behaviour of the wind gusts. In this work, the scale of turbulence, $L_{u}$, and $L_{v}$ are assigned constant values of $L_{u}=L_{v}=722.5 \mathrm{~m}$. And for low altitude region (altitude $<1000 \mathrm{ft}$ ) the $\sigma_{u}, \sigma_{v}$, and $\sigma_{w}$ turbulence intensities are given by

$$
\begin{gathered}
\frac{\sigma_{w}=0.1 W_{20}}{\sigma_{w}}=\frac{\sigma_{v}}{\sigma_{w}}=\frac{1}{(0.177+0.000823 h)^{0.4}}
\end{gathered}
$$

where, $W_{20}$ is the wind speed at $20 \mathrm{ft}(6 \mathrm{~m})$ above the ground and can be approximated by $U$ and altitude is described by $h$. In this paper, we consider a typical level of wind speed is 10 $\mathrm{m} / \mathrm{s}$ and altitude is $0.5 \mathrm{~m}$.

\section{Controller Design}

In this section, a robust adaptive bakcstepping controller is designed to keep the altitude of a small-scale helicopter in a desired way. The vertical dynamics is dependent on the altitude $z$ and the collective pitch, $\theta_{c}$. Again, the altitude $z$ of helicopter is controlled by $T_{m r}$ through the collective pitch, $\theta_{c}$. So, vertical dynamics motion equations of the helicopter are rewritten as follows:

$$
\dot{z}=w
$$

$$
\dot{w}=\frac{m g-T_{m r}}{m}+A
$$

where, A corresponds to unknown atmospheric disturbances. Step 1: The design process starts with the definition of the altitude tracking error and its derivative as follows:

$$
e_{1}=z-z_{0}, \dot{e}_{1}=\dot{z}, \quad \dot{e}_{1}=v_{z}
$$

where, $z_{0}$ is the desired altitude. We view $v_{z}$ as a virtual control law for equation (15) and let $e_{2}$ be an error variable representing the difference between the actual and virtual control of (15) i.e.,

$$
\begin{aligned}
& e_{2}=v_{z}-v_{z d} \\
& v_{z}=e_{2}+v_{z d}
\end{aligned}
$$

Therefore, $\dot{e}_{1}=e_{2}+v_{z d}$

In this step our control objective is to design a virtual control law $v_{z d}$ which make $e_{1} \rightarrow 0$. Now consider a control Lyapunov function,

$$
W_{1}=\frac{1}{2} e_{1}^{2}
$$

And its derivative as follows,

$$
\begin{aligned}
& \dot{W}_{1}=e_{1} \dot{e}_{1} \\
& \dot{W}_{1}=e_{1}\left(e_{2}+v_{z d}\right)
\end{aligned}
$$

We can now select an appropriate virtual control $v_{z d}$ which would make $\dot{W}_{1} \leq 0$. A possible choice is

$$
v_{z d}=-\alpha e_{1}
$$

that makes

$$
\dot{W}_{1}(z) \leq 0
$$

where, $\alpha$ is a scalar parameter which can be used to tune the output response.

And $\dot{W}_{1}=-\alpha e^{2}{ }_{1}+e_{1} e_{2}$

Clearly if $e_{2}=0$ then $\dot{W}_{1}=-\alpha e^{2}{ }_{1} \leq 0$

Now time derivative of the equation (17) as follows,

$$
\begin{aligned}
& \dot{v}_{z d}=-\alpha \dot{e}_{1} \\
& \dot{v}_{z d}=-\alpha v_{z}
\end{aligned}
$$

Step 2: We derive the error dynamics for $e_{2}=v_{z}-v_{z d}$ and its time derivative as follows,

$$
\begin{aligned}
& \dot{e}_{2}=\dot{v}_{z}-\dot{v}_{z d} \\
& \dot{e}_{2}=g-\frac{T_{m r}}{m}+A+\alpha v_{z}
\end{aligned}
$$

Again we know,

$$
T_{m r}=\frac{\rho a(\Omega R)^{2} A_{b}}{2}\left[\frac{\theta_{0}}{3}\left(1+\frac{3}{2} \mu^{2}\right)-\frac{1}{2} \lambda^{\prime}\right]
$$


where, advance ratio, $\mu=\frac{v_{t}}{\Omega R}$,

$$
\begin{gathered}
v_{n}=\left(a_{1}+i_{s}\right) u-b_{1} v-v_{z}, \\
v_{t}^{2}=u^{2}+v^{2}, \\
v_{i}=\frac{\eta_{G E} T_{m r}}{2 \pi R^{2} \rho \sqrt{u^{2}+v^{2}+\left(w-v_{i}\right)^{2}}}, \\
\eta_{G E}=\frac{K_{G E}}{1+\left|\frac{2 R}{h}\right|^{2}}
\end{gathered}
$$

where, $\eta_{G E}$ is the reduction $h$ of induced velocity due to ground effect and $K_{G E}=\frac{2 h}{R}$ [14] setting the magnitude
of the ground effect.

Now by substituting the value of $T_{m r}$ into equation (19) we get,

$$
\dot{e}_{2}=g-\frac{B \theta_{0}}{3 m}\left(1+\frac{3}{2} \mu^{2}\right)+\frac{B}{2 m} \lambda^{\prime}+A+\alpha v_{z}
$$

where, $B=\frac{\rho a(\Omega R)^{2} A_{b}}{2}$.

In the above equation the actual control input appears. Our objective is to design the actual control input $\theta_{0}$ such that $e_{1}$ and $e_{2}$ converge to zero. Now choose a Lyapunov function $W_{2}$ as follows,

$$
W_{2}=W_{1}+\frac{1}{2} e_{2}^{2}
$$

And its time derivative as follows,

$$
\begin{aligned}
\dot{W}_{2}= & \dot{W}_{1}+e_{2} \dot{e}_{2} \\
\dot{W}_{2}= & -\alpha e_{1}^{2}+e_{1} e_{2}+e_{2}\left\{g-\frac{B \theta_{0}}{3 m}\left(1+\frac{3}{2} \mu^{2}\right)+\frac{B}{2 m} \lambda^{\prime}\right. \\
& \left.+A+\alpha v_{z}\right\} \\
\dot{W}_{2}= & -\alpha e_{1}^{2}+e_{2}\left\{e_{1}+g-\frac{B \theta_{0}}{3 m}\left(1+\frac{3}{2} \mu^{2}\right)+\frac{B}{2 m} \lambda^{\prime}\right. \\
& \left.+A+\alpha v_{z}\right\}
\end{aligned}
$$

The derivative will satisfy

$$
\dot{W}_{2}=-\alpha e_{1}^{2}-\beta e_{2}^{2}
$$

when the control input is

$$
\theta_{0}=\frac{3 m}{B\left(1+\frac{3}{2} \mu^{2}\right)}\left[e_{1}+g+\frac{B}{2 m} \lambda^{\prime}+A+\alpha v_{z}+\beta e_{2}\right]
$$

In the subsequence derivation procedure, unless otherwise specified, the notations are defined the same as that for the adaptive backstepping controller. Now the design robust adaptive collective pitch control is

$$
\theta_{0}=\frac{3 m}{B\left(1+\frac{3}{2} \mu^{2}\right)}\left[e_{1}+g+\frac{B}{2 m} \lambda^{\prime}+\hat{A}+\alpha v_{z}+\beta e_{2}+\eta_{\theta}\right]
$$

where, $\hat{A}$ is an estimate parameter which represents a 'best-guess' for the unknown parameter $A$ and $\eta_{\theta}$ is a robust controller used to compensate for the mismatching between $A$ and $\hat{A}$.

The robust controller $\eta_{\theta}$ is designed as

$$
\eta_{\theta}=\rho_{\theta} e_{2}
$$

$\rho_{\theta}$ is a positive scalar function defined as the following

$$
\rho_{\theta}=(|z|) \widetilde{A}_{\theta b}
$$

$\widetilde{A}_{\theta b}$ is a bounding parameter selected according to

$$
\widetilde{A}_{\theta b} \geq|\widetilde{A}|=|\hat{A}-A|
$$

After this selection by substituting (26) into (19) the derivative of the vertical velocity tracking error is

$$
\dot{e}_{2}=-e_{1}-\beta e_{2}-\widetilde{A}-\eta_{\theta}
$$

Again, consider a Lyapunov function which is used to augment the estimated parameter error,

$$
W_{3}=W_{1}+\frac{1}{2} e_{2}^{2}+\frac{1}{2 \gamma} \widetilde{A}^{2}
$$

And its time derivative as follows,

$$
\begin{aligned}
& \dot{W}_{3}=\dot{W}_{1}+e_{2} \dot{e}_{2}+\frac{1}{\gamma} \widetilde{A} \frac{d \hat{A}}{d t} \\
& \dot{W}_{3}=-\alpha e_{1}^{2}+e_{1} e_{2}+e_{2}\left(-e_{1}-\beta e_{2}-\tilde{A}-\eta_{\theta}\right)+\frac{1}{\gamma} \tilde{A} \frac{d \hat{A}}{d t} \\
& \dot{W}_{3}=-\alpha e_{1}^{2}-\beta e_{2}^{2}-\rho_{\theta} e_{2}^{2}-\widetilde{A}\left(e_{2}-\frac{1}{\gamma} \frac{d \hat{A}}{d t}\right)
\end{aligned}
$$

where, $\gamma$ is a positive design constant that determines the convergence speed of the estimate. In order to render the non-positivity of the Lyapunov derivative of the above equation, we will choose the estimated parameter $A$ and $A$ as follows,

$$
\frac{d \hat{A}}{d t}=\gamma e_{2} \text { thus } \dot{W}_{3}=-\alpha e_{1}^{2}-\beta e_{2}^{2}-\rho_{\theta} e_{2}^{2} \leq 0 .
$$


By the definition of the Lyapunov function and its non-positive derivative, the altitude tracking error $e_{l}$, the vertical velocity tracking error $e_{2}$, and the estimated parameter error are bouneded signal. Thus the derivative of the error signals are also bounded.

\section{Simulation Result}

In this section, simulation results are presented to investigate the performance of the proposed robust adaptive backstepping controller for altitude control of the small scale helicopter based on simulation parameters consistent with those employed in real applications. Operation limits in the collective pitch $\left(1^{0}<\theta_{\text {col }}<10^{\circ}\right)$ and the rate limit in servo dynamics $\left(\left|\dot{\theta}_{c o l}\right|<20^{\circ} / \mathrm{s}\right)$, are taken into account in the simulation model. The horizontal wind disturbance is shown in Fig.1. The simulation result of the robust adaptive backstepping controller with ground effect compensation is depicted in Fig.2. It is clear that altitude tracking performance is improved by compensating the ground effect, while Fig. 3 indicates that large deviation appears at the altitude tracking without ground effect compensation near ground surface. The linear velocity in the $z$ direction is slightly fluctuating as illustrated in Fig.4.

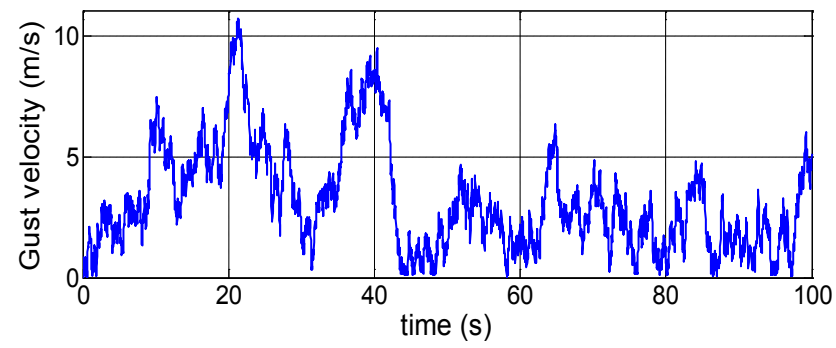

Figure 1. Wind disturbance to test controller

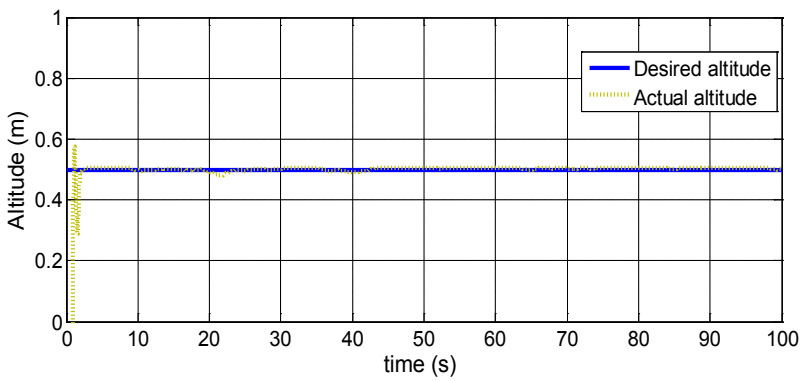

Figure 2. Behaviour of altitude with ground effect compensation

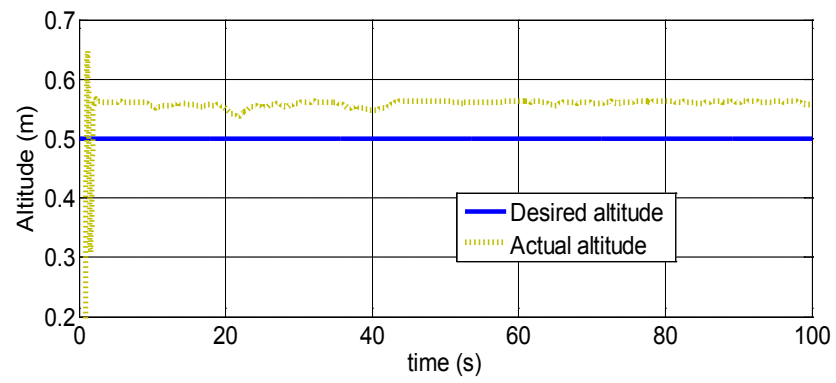

Figure 3. Behaviour of altitude without ground effect compensation

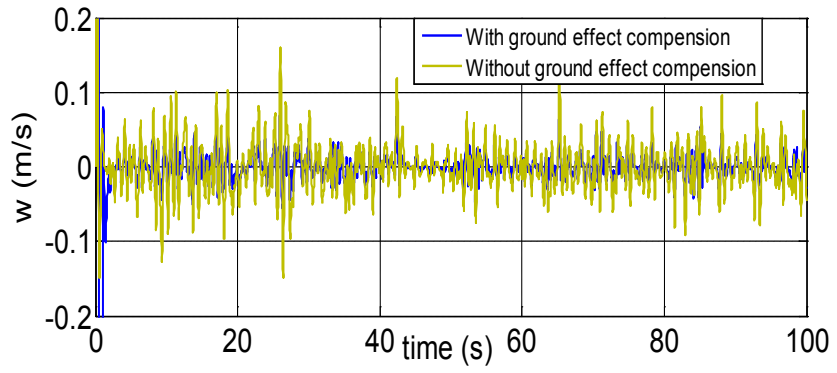

Figure 4. Vertical velocity response

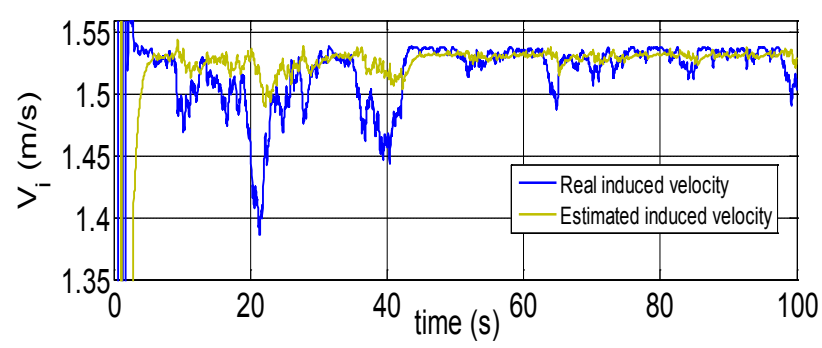

Figure 5. Estimation of induced velocity

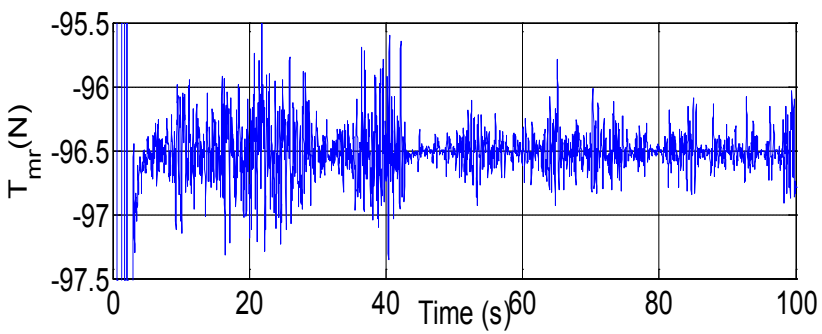

Figure 6. Main rotor thrust

Fig. 5 shows that the estimated induced velocity is very close to the real induced velocity, with maximum estimation error of $0.1 \mathrm{~m} / \mathrm{s}$ in the presence of ground effect. Fig.6 represents the variation in hovering thrust due to ground effect and external wind gusts near ground surface. The control input obtained using the robust adaptive backstepping controller is shown in Fig.7. It doesn't exceed the constraints for altitude control of a small helicopter. It can effectively compensate the ground effect as well as the external wind gusts effect, and the UAH can hover near ground surface.

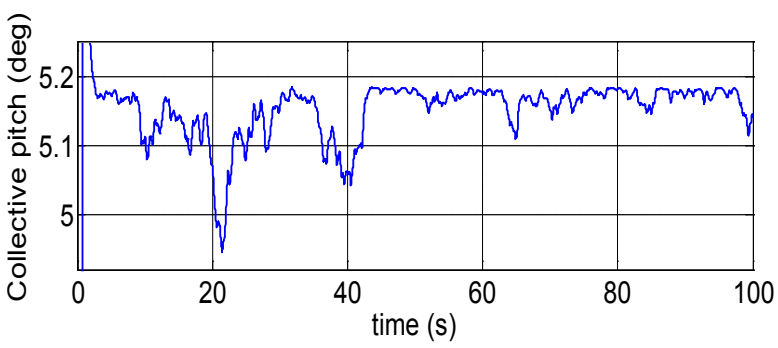

Figure 7. Control input

\section{Conclusion}

In this paper, a nonlinear robust adaptive backstepping 
control scheme for the altitude control of a small scale helicopter near ground surface based on control Lyapunov approach is demonstrated. The algorithm controls the altitude dynamics of a VARIO small-scale helicopter. The simulation results clearly investigated the robustness of the proposed controller by tracking the altitude of the helicopter in the presence of ground effect and external wind gusts. Future works will deal with the implementation of the proposed autonomous flight control method on the real system and flight test to prove its feasibility in real applications.

\section{REFERENCES}

[1] Xiaodong Wang and Xiaoguang Zhao, "A practical survey on the flight control system of small-scale unmanned helicopter", 7th World Congress on Intelligent Control and Automation (WCICA 2008), pp.364-369, 2008.

[2] Adnan Martini, François Léonard, and Gabriel Abba, "Dynamic Modelling and Stability Analysis of Model-Scale Helicopters Under Wind Gust", J Intell Robot Syst (2009) 54:647-686.

[3] Xilin Yang, Hemanshu Pota, and Matt Garratt, "Design of a Gust Attenuation Controller for Landing Operations of Unmanned Autonomous Helicopters", 18th IEEE International Conference on Control Applications, July 2009.

[4] Kumeresan A. Danapalasingam, John-Josef Leth, Anders la Cour-Harbo and Morten Bisgaard, "Robust Helicopter Stabilization in the Face of Wind Disturbance", 49th IEEE Conference on Decision and Control, December 15-17, 2010 Hilton Atlanta Hotel, Atlanta, GA, USA.

[5] H. Wang, A. A. Mian, D. Wang, and H. Duan, "Robust multi-mode flight control design for an unmanned helicopter based on multi-loop structure", International Journal of Control, Automation, and Systems 7 (2009), 723-730.

[6] T. Cheviron, F. Plestan, and A. Chriette, "A robust guidance and control scheme of an autonomous scale helicopter in presence of wind gusts", International Journal of Control, Vol. 82, No. 12 December 2009, 2206-2220.

[7] K. P. Tee, S. S. Ge, and F. E. H. Tay, "Adaptive neural network control for helicopter in vertical flight", IEEE Transaction on Control System and Technology, Vol.16, No.4, pp.753-762, 2008.
[8] Alejandro Dzul, Rogelio Lozano and Pedro Castillo, "Adaptive Altitude Control for a Small Helicopter in a Vertical Flying Stand", Proceedings of the 42nd IEEE Conference on Decision and Control Maui, Hawaii USA, December 2003

[9] Keng Peng Tee, Shuzhi Sam Ge, and Francis E. H. Tay, "Adaptive Neural Network Control for Helicopters in Vertical Flight", IEEE Transactions on Control Systems Technology, Vol. 16, No. 4, July 2008.

[10] Jongkwon Kim, Soohong Park, and Cheolsoon Jang, "Control System design for an Unmanned Helicopter", http://www.aciar2005.ait.ac.th/CD/F-27.pdf (cited on 13th of September, 2011)

[11] J. Kaloust, C. Ham, and Z. Qu, "Nonlinear autopilot control design for a 2-DOF helicopter model", IEE Proc., Control Theory Appl. -- Volume 144, Issue 6, p.612-616, November 1997.

[12] Kenichiro Nonaka and Hirokazu Sugizaki, "Integral Sliding Mode Altitude Control for a Small Model Helicopter with Ground Effect Compensation," American Control Conference on O'Farrell Street, San Francisco, CA, USA June 29 - July 01, 2011

[13] Tushar. K. Roy, Matt Garratt, Hemanshu R. Pota and Mahendra Kumar Samal, "Robust Altitude Control for a Small Helicopter by Considering the Ground Effect Compensation," The 10th World Congress on Intelligent Control and Automation (WCICA), pp. 1796-1800, July 6-8, Beijing, China, 2012.

[14] J. Gordon Leishman, "Principles of Helicopter Aerodynamics", Second edition, 2006.

[15] Tushar. K. Roy, Matt Garratt, Hemanshu R. Pota and Hamid Teimoori, "Robust Altitude Control of an Unmanned Autonomous Helicopter Using Backstepping," IEEE The 10th World Congress on Intelligent Control and Automation (WCICA), pp. 1650-1654, July 6-8, Beijing, China, 2012.

[16] T. K. Roy and M. Garratt , "Altitude Control of an Unmanned Autonomous Helicopter via Robust Backstepping Controller under Horizontal Wind Gusts," IEEE 7th International Conference on Electrical and Computer Engineering (ICECE' 2012), pp. 771-774, December 20-22, 2012, Dhaka, Bangladesh.

[17] T. K. Roy and H. M. Rasel, "Altitude Control of an Unmanned Autonomous Helicopter via Adaptive Backstepping Controller Under Horizontal Wind Gusts," International Journal of Computer and Electronics Engineering, Vol. 5, No. 1, pp. 1-7, ISSN : 0975-4202, India, February 2013. 